\title{
Saari's homographic conjecture for planar equal-mass three-body problem under a strong force potential
}

\author{
Toshiaki Fujiwara $^{1}$, Hiroshi Fukuda ${ }^{2}$, Hiroshi Ozaki ${ }^{3}$ \\ and Tetsuya Taniguchi ${ }^{4}$ \\ 1,2,4 College of Liberal Arts and Sciences, Kitasato University, 1-15-1 Kitasato, \\ Sagamihara, Kanagawa 252-0329, Japan \\ 3 General Education Program Center, Tokai University, Shimizu Campus, \\ 3-20-1, Orido, Shimizu, Shizuoka 424-8610, Japan \\ E-mail: ${ }^{1}$ fujiwara@kitasato-u.ac.jp, ${ }^{2}$ fukuda@kitasato-u.ac.jp, \\ ${ }^{3}$ ozaki@tokai-u.jp, ${ }^{4}$ tetsuya@kitasato-u.ac.jp
}

\begin{abstract}
Donald Saari conjectured that the $N$-body motion with constant configurational measure is a motion with fixed shape. Here, the configurational measure $\mu$ is a scale invariant product of the moment of inertia $I=\sum_{k} m_{k}\left|q_{k}\right|^{2}$ and the potential function $U=\sum_{i<j} m_{i} m_{j} /\left|q_{i}-q_{j}\right|^{\alpha}, \alpha>0$. Namely, $\mu=I^{\alpha / 2} U$. We will show that this conjecture is true for planar equal-mass three-body problem under the strong force potential $\sum_{i<j} 1 /\left|q_{i}-q_{j}\right|^{2}$.
\end{abstract}

PACS numbers: 45.20.D-, 45.20.Jj, 45.50.Jf

Submitted to: J. Phys. A: Math. Gen. 


\section{Saari's homographic conjecture}

In 1969, Donald Saari conjectured that if a $N$-body system has a constant moment of inertia then the motion is a rotation with constant mutual distances $r_{i j}$ [ . Here, the moment of inertia $I$ is defined by

$$
I=\sum_{k} m_{k}\left|q_{k}\right|^{2},
$$

with $m_{k}$ and $q_{k}$ being the masses and position vectors of body $k=1,2,3, \ldots N$. This is now called Saari's original conjecture. In the conference "Saarifest 2005" at Guanajuato Mexico, Richard Moeckel proved that the original conjecture is true for three-body problem in $\mathbb{R}^{d}$ for any $d \geq 2$ [4, 5].

In the same conference, Saari extended his conjecture. His new conjecture is "if the configurational measure $I^{\alpha / 2} U$ is constant then the $N$-body motion is homographic" 9, 10, where,

$$
U=\sum_{i<j} \frac{m_{i} m_{j}}{r_{i j}^{\alpha}}
$$

is the potential function. This is indeed a natural extension of the original conjecture. Note that a solution $q_{k}$ of $N$ bodies is called homographic if the configuration formed by the $N$ bodies moves in such a way as to remain similar to itself. For $\alpha \neq 2$, we can show that if the moment of inertia is constant then $U$ is constant, therefore the configurational measure is also constant. For $\alpha=2$, on the other hand, $I=$ constant does not yields $U=$ constant [1]. Actually, there are some counter examples for the original conjecture for $\alpha=2$ [2] [7. However, the extended conjecture is expected to be true for $\alpha=2$ and all $\alpha>0$.

Florin Diacu, Toshiaki Fujiwara, Ernesto Pérez-Chavela and Manuele Santoprete called this conjecture the "Saari's homographic conjecture" and partly proved this conjecture for some cases [3]. No one proved this conjecture completely, as far as we know.

Obviously, Saari's conjecture is related to the motion in shape. Here, a shape is a configuration of $N$ bodies up to rotation and scaling. To prove the Saari's original and homographic conjecture, it is important to find appropriate variables to describe motion in shape. The moment of inertia $I$ describes the motion in size, and the angular momentum $C$ describes the rotation. What are the appropriate variables to describe the motion in shape?

An answer was given by Richard Moeckel and Richard Montgomery [6. They used the ratio of the Jacobi coordinate to describe the motion in shape for planar three-body problem. Let us explain precisely. To avoid non-essential complexity, let us consider equal masses case, and set $m_{k}=1$ in this paper. We take the center of mass frame. So, we have

$$
\sum_{k} q_{k}=0 .
$$

In the three-body problem, we have two Jacobi coordinates,

$$
\begin{aligned}
& z_{1}=q_{2}-q_{1}, \\
& z_{2}=q_{3}-\frac{q_{1}+q_{2}}{2}=\frac{3}{2} q_{3} .
\end{aligned}
$$


Since, we are considering planar motions, let us identify $q_{k}$ and $z_{i}$ with complex numbers. Then, we can define the ratio of the Jacobi coordinates,

$$
\zeta=\frac{z_{2}}{z_{1}}=\frac{3}{2} \frac{q_{3}}{q_{2}-q_{1}} .
$$

Note that the variable $\zeta$ is invariant under the size change and rotation, $q_{k} \mapsto \lambda e^{i \theta} q_{k}$ with $\lambda, \theta \in \mathbb{R}$. Therefore, $\zeta$ depends only on the shape. The great idea by Moeckel and Montgomery is to use the variable $\zeta$ to describe the shape. They actually write down the Lagrangian by the variable $\zeta$, the moment of inertia $I$ and the rotation angle $\theta$. They also write down the equations of motion for these variables.

Using the formulation developed by Moeckel and Montgomery, we will show that the Saari's homographic conjecture is true for planar equal-mass three-body problem under the strong force potential,

$$
U=\sum_{i<j} \frac{1}{\left|q_{i}-q_{j}\right|^{2}}
$$

Namely, we will show that $d \zeta / d t=0$ if and only if $I U=$ constant.

In the section 2, we derive the Lagrangian in terms of $I, \theta$ and $\zeta$ by elementary calculations. The equations of motion and some useful relations are also shown in this section. Every relations in the section 2 are valid for $\alpha \neq 0$. In the section 3. we concentrate on the strong force potential $\alpha=2$. We will prove the Saari's homographic conjecture for this case. Details in calculation are shown in Appendix A. In the Appendix B, some properties of the shape variable $\zeta$, which may useful to understand this variable.

\section{Lagrangian for planar equal-mass three-body problem in terms of shape, size and rotation angle}

In this section, we consider the planar equal-mass three-body problem under the potential function (2) with $m_{1}=m_{2}=m_{3}=1$ for $\alpha \neq 0$. Let $K=\sum_{k}\left|d q_{k} / d t\right|^{2}$ be twice of the kinetic energy, and let the Lagrangian and the total energy be $L=K / 2+U / \alpha$ and $E=K / 2-U / \alpha$, respectively. In the center of mass frame (3) all quantities $\xi_{k}=q_{k} /\left(q_{2}-q_{1}\right)$ are expressed by the shape variable in (6), as follows,

$$
\begin{aligned}
& \xi_{1}=\frac{q_{1}}{q_{2}-q_{1}}=-\frac{1}{2}-\frac{\zeta}{3}, \\
& \xi_{2}=\frac{q_{2}}{q_{2}-q_{1}}=+\frac{1}{2}-\frac{\zeta}{3}, \\
& \xi_{3}=\frac{q_{3}}{q_{2}-q_{1}}=\frac{2}{3} \zeta .
\end{aligned}
$$

Obviously, the triangle made of $q_{1}, q_{2}, q_{3}$ is similar to the triangle made of $\xi_{1}, \xi_{2}, \xi_{3}$. Therefore, there are some $I \geq 0$ and $\theta \in \mathbb{R}$, such that

$$
q_{k}=\sqrt{I} e^{i \theta} \frac{\xi_{k}}{\sqrt{\sum_{l}\left|\xi_{\ell}\right|^{2}}} .
$$

We treat $I, \theta$ and $\zeta$ as independent dynamical variables. 


\subsection{Lagrangian}

Then, direct calculations for $K$ yields

$$
\begin{aligned}
K & =\sum_{k}\left|\frac{\dot{I}}{2 \sqrt{I}} \frac{\xi_{k}}{\sqrt{\sum_{l}\left|\xi_{\ell}\right|^{2}}}+i \dot{\theta} \sqrt{I} \frac{\xi_{k}}{\sqrt{\sum_{l}\left|\xi_{\ell}\right|^{2}}}+\sqrt{I} \frac{d}{d t}\left(\frac{\xi_{k}}{\sqrt{\sum_{l}\left|\xi_{\ell}\right|^{2}}}\right)\right|^{2} \\
& =\frac{\dot{I}^{2}}{4 I}+I\left(\dot{\theta}+\frac{\frac{2}{3} \zeta \wedge \dot{\zeta}}{\frac{1}{2}+\frac{2}{3}|\zeta|^{2}}\right)^{2}+\frac{I}{3} \frac{|\dot{\zeta}|^{2}}{\left(\frac{1}{2}+\frac{2}{3}|\zeta|^{2}\right)^{2}} .
\end{aligned}
$$

Here, the wedge product $\wedge$ represents $(a+i b) \wedge(c+i d)=a d-b c$ for $a, b, c, d \in \mathbb{R}$, and the $\operatorname{dot} d / d t$. On the other hand, the potential function $U$ is

$$
\begin{aligned}
U & =\frac{1}{I^{\alpha / 2}}\left(\sum_{k}\left|\xi_{k}\right|^{2}\right)^{\alpha / 2} \sum_{i<j} \frac{1}{\left|\xi_{i}-\xi_{j}\right|^{\alpha}} \\
& =\frac{1}{I^{\alpha / 2}}\left(\frac{1}{2}+\frac{2}{3}|\zeta|^{2}\right)^{\alpha / 2}\left(1+\frac{1}{|\zeta-1 / 2|^{\alpha}}+\frac{1}{|\zeta+1 / 2|^{\alpha}}\right) .
\end{aligned}
$$

Therefore, the configurational measure $\mu$ is a function of the shape variable $\zeta$,

$$
\mu(\zeta)=I^{\alpha / 2} U=\left(\frac{1}{2}+\frac{2}{3}|\zeta|^{2}\right)^{\alpha / 2}\left(1+\frac{1}{|\zeta-1 / 2|^{\alpha}}+\frac{1}{|\zeta+1 / 2|^{\alpha}}\right)
$$

Thus, we get the Lagrangian

$$
L=\frac{\dot{I}^{2}}{8 I}+\frac{I}{2}\left(\dot{\theta}+\frac{\frac{2}{3} \zeta \wedge \dot{\zeta}}{\frac{1}{2}+\frac{2}{3}|\zeta|^{2}}\right)^{2}+\frac{I}{6} \frac{|\dot{\zeta}|^{2}}{\left(\frac{1}{2}+\frac{2}{3}|\zeta|^{2}\right)^{2}}+\frac{\mu(\zeta)}{\alpha I^{\alpha / 2}},
$$

in terms of $I, \theta, \zeta$ and their velocities. Or, identifying $\zeta=x+i y$ with $x, y \in \mathbb{R}$ to a two dimensional vector $\mathbf{x}=(x, y)$, the Lagrangian is expressed as

$$
L=\frac{\dot{I}^{2}}{8 I}+\frac{I}{2}\left(\dot{\theta}+\frac{\frac{2}{3} \mathbf{x} \wedge \dot{\mathbf{x}}}{\frac{1}{2}+\frac{2}{3}|\mathbf{x}|^{2}}\right)^{2}+\frac{I}{6} \frac{|\dot{\mathbf{x}}|^{2}}{\left(\frac{1}{2}+\frac{2}{3}|\mathbf{x}|^{2}\right)^{2}}+\frac{\mu(\mathbf{x})}{\alpha I^{\alpha / 2}}
$$

with $\mathbf{x} \wedge \dot{\mathbf{x}}=x \dot{y}-y \dot{x}$ and

$$
\mu(\mathbf{x})=\left(\frac{1}{2}+\frac{2}{3}|\mathbf{x}|^{2}\right)^{\alpha / 2}\left(1+\frac{1}{\left((x-1 / 2)^{2}+y^{2}\right)^{\alpha / 2}}+\frac{1}{\left((x+1 / 2)^{2}+y^{2}\right)^{\alpha / 2}}\right) .
$$

\subsection{Equation of motion for rotation angle}

Obviously, the variable $\theta$ is cyclic. Therefore, we get the conservation law of the angular momentum,

$$
C=\frac{\partial L}{\partial \dot{\theta}}=I\left(\dot{\theta}+\frac{\frac{2}{3} \mathbf{x} \wedge \dot{\mathbf{x}}}{\frac{1}{2}+\frac{2}{3}|\mathbf{x}|^{2}}\right)=\text { constant. }
$$

Then, the kinetic energy $K / 2$ is given by

$$
\frac{K}{2}=\frac{\dot{I}^{2}}{8 I}+\frac{C^{2}}{2 I}+\frac{I}{6} \frac{|\dot{\mathbf{x}}|^{2}}{\left(\frac{1}{2}+\frac{2}{3}|\mathbf{x}|^{2}\right)^{2}} .
$$

The three terms in the right hand side represent kinetic energies for the scale change, for the rotation and for the shape change. 


\subsection{Equation of motion for the moment of inertia}

The Euler-Lagrange equation for the moment of inertia $I$,

$$
\frac{d}{d t}\left(\frac{\partial L}{\partial \dot{I}}\right)-\frac{\partial L}{\partial I}=0
$$

yields

$$
\begin{aligned}
\frac{\ddot{I}}{4} & =\frac{\dot{I}^{2}}{8 I}+\frac{C^{2}}{2 I}+\frac{I}{6} \frac{|\dot{\mathbf{x}}|^{2}}{\left(\frac{1}{2}+\frac{2}{3}|\mathbf{x}|^{2}\right)^{2}}-\frac{1}{2} \frac{\mu(\mathbf{x})}{I^{\alpha / 2}} \\
& =E+\left(\frac{1}{\alpha}-\frac{1}{2}\right) U .
\end{aligned}
$$

Multiplying $\dot{I}$ both side of the equation 22, we get

$$
-\frac{\mu}{\alpha}(1-\alpha / 2) I^{-\alpha / 2} \dot{I}=E \dot{I}-\frac{1}{4} \dot{I} \ddot{I} \text {. }
$$

This means

$$
\begin{aligned}
-\frac{\mu}{\alpha} \frac{d}{d t}\left(I^{1-\alpha / 2}\right) & =\frac{d}{d t}\left(E I-\frac{\dot{I}^{2}}{8}\right) \\
& =\frac{d}{d t}\left(\frac{C^{2}}{2}+\frac{I^{2}}{6} \frac{|\dot{\mathbf{x}}|^{2}}{\left(\frac{1}{2}+\frac{2}{3}|\mathbf{x}|^{2}\right)^{2}}-\frac{\mu}{\alpha} I^{1-\alpha / 2}\right) .
\end{aligned}
$$

Therefore, we get

$$
\frac{d}{d t}\left(\frac{I^{2}}{6} \frac{|\dot{\mathbf{x}}|^{2}}{\left(\frac{1}{2}+\frac{2}{3}|\mathbf{x}|^{2}\right)^{2}}\right)=\frac{I^{1-\alpha / 2}}{\alpha} \frac{d \mu}{d t} .
$$

This relation was first derived by Saari [9]. We would like to call this "Saari's relation". Inspired by this relation, let us introduce new 'time' variable $s$ defined by

$$
d s=\frac{1}{I}\left(\frac{1}{2}+\frac{2}{3}|\mathbf{x}|^{2}\right) d t .
$$

Then, we have

$$
\frac{d}{d t}=\frac{1}{I}\left(\frac{1}{2}+\frac{2}{3}|\mathbf{x}|^{2}\right) \frac{d}{d s}
$$

and the Saari's relation 26 is

$$
\frac{d}{d s}\left(\frac{1}{6}\left|\frac{d \mathbf{x}}{d s}\right|^{2}\right)=\frac{I^{1-\alpha / 2}}{\alpha} \frac{d \mu}{d s} .
$$

2.4. Equation of motion for the shape variables

The Euler-Lagrange equation for $\mathbf{x}$,

$$
\frac{d}{d t}\left(\frac{\partial L}{\partial \dot{\mathbf{x}}}\right)-\frac{\partial L}{\partial \mathbf{x}}=0
$$


yields

$$
\begin{aligned}
& \frac{d}{d t}\left(\frac{I}{3\left(1 / 2+2|\mathbf{x}|^{2} / 3\right)^{2}} \frac{d \mathbf{x}}{d t}-\frac{2 C}{3} \frac{1}{\left(1 / 2+2|\mathbf{x}|^{2} / 3\right)}(y,-x)\right) \\
& =\frac{2 C}{3} \frac{1}{\left(1 / 2+2|\mathbf{x}|^{2} / 3\right)} \frac{d}{d t}(y,-x)+\frac{2 C}{3}\left(\mathbf{x} \wedge \frac{d \mathbf{x}}{d t}\right) \frac{\partial}{\partial \mathbf{x}}\left(\frac{1}{\left(1 / 2+2|\mathbf{x}|^{2} / 3\right)}\right) \\
& +\frac{I}{6}\left|\frac{d \mathbf{x}}{d t}\right|^{2} \frac{\partial}{\partial \mathbf{x}}\left(\frac{1}{\left(1 / 2+2|\mathbf{x}|^{2} / 3\right)^{2}}\right)+\frac{1}{\alpha I^{\alpha / 2}} \frac{\partial \mu}{\partial \mathbf{x}} .
\end{aligned}
$$

Using the 'time' variable $s$, this equation of motion is

$$
\frac{d^{2} \mathbf{x}}{d s^{2}}=\frac{2 C-\frac{4}{3}\left(\mathbf{x} \wedge \frac{d \mathbf{x}}{d s}\right)}{\frac{1}{2}+\frac{2}{3}|\mathbf{x}|^{2}}\left(\frac{d y}{d s},-\frac{d x}{d s}\right)+\frac{3 I^{1-\alpha / 2}}{\alpha} \frac{\partial \mu}{\partial \mathbf{x}} .
$$

Inner product of $d \mathbf{x} / d s$ and $d^{2} \mathbf{x} / d s^{2}$ yields

$$
\frac{d \mathbf{x}}{d s} \cdot \frac{d^{2} \mathbf{x}}{d s^{2}}=\frac{3 I^{1-\alpha / 2}}{\alpha} \frac{d \mathbf{x}}{d s} \cdot \frac{\partial \mu}{\partial \mathbf{x}}=\frac{3 I^{1-\alpha / 2}}{\alpha} \frac{d \mu}{d s} .
$$

This is nothing but the Saari's relation in (29). While, the wedge product of the same pair yields

$$
\frac{d \mathbf{x}}{d s} \wedge \frac{d^{2} \mathbf{x}}{d s^{2}}=-\frac{2 C-\frac{4}{3}\left(\mathbf{x} \wedge \frac{d \mathbf{x}}{d s}\right)}{\frac{1}{2}+\frac{2}{3}|\mathbf{x}|^{2}}\left|\frac{d \mathbf{x}}{d s}\right|^{2}+\frac{3 I^{1-\alpha / 2}}{\alpha} \frac{d \mathbf{x}}{d s} \wedge \frac{\partial \mu}{\partial \mathbf{x}} .
$$

Every equations are valid for all $\alpha \neq 0$. We will use these expressions later.

\section{Proof of the Saari's homographic conjecture under the strong force potential}

In this section, we will prove the Saari's homographic conjecture for the case $\alpha=2$, namely, $\dot{\zeta}=0$ if and only if $\mu=$ constant .

Let us assume

$$
\mu=\mu_{0}=\text { constant. }
$$

Then, by the Saari's relation 29 , we have

$$
\left|\frac{d \mathbf{x}}{d s}\right|^{2}=k^{2}
$$

with constant $k \geq 0$.

If $k=0$, then $d \mathbf{x} / d s=0$, namely $\dot{\zeta}=0$.

Let us examine the case $k>0$. For this case, the point $\mathbf{x}(s)$ moves on the curve $\mu(\mathbf{x})=\mu_{0}$ with constant speed $|d \mathbf{x} / d s|=k$. This motion of $\mathbf{x}(s)$ is not able to keep $\partial \mu / \partial \mathbf{x}=0$. Because, the points that satisfy $\partial \mu / \partial \mathbf{x}=0$ are only five central configurations at $\mathbf{x}=( \pm 3 / 2,0),(0,0),(0, \pm \sqrt{3} / 2)$. See figure 1 .

So, we can take some finite arc of the curve $\mu(\mathbf{x})=\mu_{0}$ on which

$$
\frac{\partial \mu}{\partial \mathbf{x}} \neq 0
$$




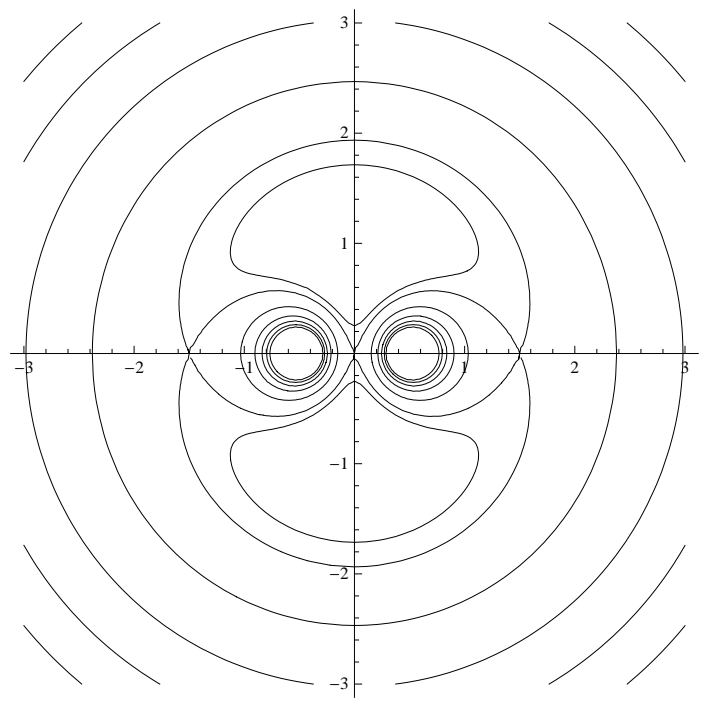

Figure 1. Curves of $\mu(x, y)=\mu_{0}$ for several $\mu_{0}$.

Then the equation (36) and

$$
\frac{d \mathbf{x}}{d s} \cdot \frac{\partial \mu}{\partial \mathbf{x}}=\frac{d \mu}{d s}=0
$$

yields

$$
\frac{d \mathbf{x}}{d s}=\frac{\epsilon k}{|\partial \mu / \partial \mathbf{x}|}\left(-\frac{\partial \mu}{\partial y}, \frac{\partial \mu}{\partial x}\right) .
$$

Here, $\epsilon= \pm 1$ determines the direction of the motion on the curve $\mu=\mu_{0}$. Differentiate this expression by $s$ again, we have

$$
\frac{d^{2} \mathbf{x}}{d s^{2}}=\frac{\epsilon k}{|\partial \mu / \partial \mathbf{x}|} \frac{d}{d s}\left(-\frac{\partial \mu}{\partial y}, \frac{\partial \mu}{\partial x}\right)+\left(-\frac{\partial \mu}{\partial y}, \frac{\partial \mu}{\partial x}\right) \frac{d}{d s}\left(\frac{\epsilon k}{|\partial \mu / \partial \mathbf{x}|}\right) .
$$

Therefore, we get

$$
\begin{aligned}
& \frac{d \mathbf{x}}{d s} \wedge \frac{d^{2} \mathbf{x}}{d s^{2}} \\
& =\frac{k^{2}}{|\partial \mu / \partial \mathbf{x}|^{2}}\left(-\frac{\partial \mu}{\partial y}, \frac{\partial \mu}{\partial x}\right) \wedge \frac{d}{d s}\left(-\frac{\partial \mu}{\partial y}, \frac{\partial \mu}{\partial x}\right) \\
& =\frac{\epsilon k^{3}}{|\partial \mu / \partial \mathbf{x}|^{3}}\left(\left(\frac{\partial \mu}{\partial y}\right)^{2} \frac{\partial^{2} \mu}{\partial x^{2}}-2 \frac{\partial \mu}{\partial x} \frac{\partial \mu}{\partial y} \frac{\partial^{2} \mu}{\partial x \partial y}+\left(\frac{\partial \mu}{\partial x}\right)^{2} \frac{\partial^{2} \mu}{\partial y^{2}}\right) .
\end{aligned}
$$

Therefore, the curvature $\rho^{-1}$ of the curve $\mu=\mu_{0}$ should be

$$
\rho^{-1}=\frac{\epsilon}{|\partial \mu / \partial \mathbf{x}|^{3}}\left(\left(\frac{\partial \mu}{\partial y}\right)^{2} \frac{\partial^{2} \mu}{\partial x^{2}}-2 \frac{\partial \mu}{\partial x} \frac{\partial \mu}{\partial y} \frac{\partial^{2} \mu}{\partial x \partial y}+\left(\frac{\partial \mu}{\partial x}\right)^{2} \frac{\partial^{2} \mu}{\partial y^{2}}\right)
$$

On the other hand, by the relation (34) which is a result of the equation of motion and the expression for the velocity (39), we have another expression for the curvature

$$
\rho^{-1}=\frac{1}{1 / 2+2|\mathbf{x}|^{2} / 3}\left(-\frac{2 C}{k}+\frac{4 \epsilon}{3|\partial \mu / \partial \mathbf{x}|} \mathbf{x} \cdot \frac{\partial \mu}{\partial \mathbf{x}}\right)-\frac{3 \epsilon}{2 k^{2}}\left|\frac{\partial \mu}{\partial \mathbf{x}}\right| .
$$


Our plan to exclude the case $k>0$ is the following. Since $|d \mathbf{x} / d s|=k=$ constant, the parameter $s$ is proportional to the arc length of the curve $\mathbf{x}(s)$ on $\mu=\mu_{0}$. Therefore, if $k>0$ there must be finite arc, on which the curvature 42 coincides with 43 . We call such arc non-Saari arc. In the following, we will show that non-Saari arc does not exist. Namely, $k>0$ is impossible.

Let us examine the condition for the two curvature have the same value. The condition is

$$
\begin{aligned}
\frac{1}{|\partial \mu / \partial \mathbf{x}|^{3}}\left(\left(\frac{\partial \mu}{\partial y}\right)^{2} \frac{\partial^{2} \mu}{\partial x^{2}}-2 \frac{\partial \mu}{\partial x} \frac{\partial \mu}{\partial y} \frac{\partial^{2} \mu}{\partial x \partial y}+\left(\frac{\partial \mu}{\partial x}\right)^{2} \frac{\partial^{2} \mu}{\partial y^{2}}\right) \\
=\frac{1}{1 / 2+2|\mathbf{x}|^{2} / 3}\left(-\frac{2 \epsilon C}{k}+\frac{4}{3|\partial \mu / \partial \mathbf{x}|} \mathbf{x} \cdot \frac{\partial \mu}{\partial \mathbf{x}}\right)-\frac{3}{2 k^{2}}\left|\frac{\partial \mu}{\partial \mathbf{x}}\right|
\end{aligned}
$$

Therefore,

$$
\begin{aligned}
\frac{4 C^{2}}{k^{2} N^{2}}|\nabla \mu|^{6}- & \left(\left(\frac{\partial \mu}{\partial y}\right)^{2} \frac{\partial^{2} \mu}{\partial x^{2}}-2 \frac{\partial \mu}{\partial x} \frac{\partial \mu}{\partial y} \frac{\partial^{2} \mu}{\partial x \partial y}+\left(\frac{\partial \mu}{\partial x}\right)^{2} \frac{\partial^{2} \mu}{\partial y^{2}}-\frac{4}{3 N}(\mathbf{x} \cdot \nabla \mu)|\nabla \mu|^{2}+\frac{3}{2 k^{2}}|\nabla \mu|^{4}\right)^{2} \\
& =0 .
\end{aligned}
$$

Where, $N=1 / 2+2|\mathbf{x}|^{2} / 3$ and $\nabla \mu=\partial \mu / \partial \mathbf{x}$. The left hand side is a ratio of polynomials of $x^{2}, y^{2}, C^{2}$ and $k^{2}$. Let the numerator of this ratio be a polynomial $P\left(x^{2}, y^{2}, C^{2}, k^{2}\right)$, then $x^{2}, y^{2}$ must satisfy the following equation,

$$
P\left(x^{2}, y^{2}, C^{2}, k^{2}\right)=0 .
$$

The maximum power of the variables for $P$ are $x^{60}, y^{60}, C^{2}$ and $k^{4}$.

On the other hand, the equation $\mu=\mu_{0}$ is also a ratio of polynomials of $x^{2}, y^{2}$ and $\mu_{0}$. Let the numerator of this ratio be a polynomial $Q$, then we have the following equation

$$
\begin{aligned}
Q\left(x^{2}, y^{2}, \mu_{0}\right)= & 27+64 x^{6}+156 y^{2}+208 y^{4}+64 y^{6} \\
& +48 x^{4}\left(3+4 y^{2}\right)+4 x^{2}\left(27+88 y^{2}+48 y^{4}\right) \\
& -6 \mu_{0}\left(16 x^{4}+8 x^{2}\left(-1+4 y^{2}\right)+\left(1+4 y^{2}\right)^{2}\right) \\
& =0 .
\end{aligned}
$$

The non-Saari arc must satisfy both $P=0$ and $Q=0$ for some value of parameters $C^{2}, k^{2}$ and $\mu_{0}$.

There is no finite arc with $x=x_{0}=$ fixed and $\mu=\mu_{0}$. Because, for $x=x_{0}, Q=0$ is a polynomial of $y^{2}$ of order $y^{6}$ with the coefficient of $y^{6}$ being $64 \neq 0$. Therefore, solutions of $y$ for $Q=0$ are discrete. Thus, any finite arc must have some finite interval $x_{1} \leq x \leq x_{2}$.

There is no finite interval $x_{1} \leq x \leq x_{2}$ that every $x$ in this interval satisfy $P=Q=0$. To show this, we eliminate $y^{2}$ from $P\left(x^{2}, y^{2}\right)=Q\left(x^{2}, y^{2}\right)=0$ to get new polynomial $R\left(x^{2}\right)=0$. This polynomial turns out to be order $x^{68}$,

$$
R=A x^{8}\left(4 x^{2}-1\right)^{6} \sum_{0 \leq n \leq 24} c_{n}\left(C^{2}, k^{2}, \mu_{0}\right) x^{2 n} .
$$

Where, $A$ is a big integer. To have continuous solution of $x$ for $R=0$, the polynomial $R$ must be identically equal to zero. Namely, all coefficients $c_{n}, n=0,1,2, \ldots, 24$ must be zero. Therefore, we have 25 conditions for only three parameters $C^{2}, k^{2}$ and $\mu_{0}$. Actually, there are no parameters to make all 25 coefficients vanish. See Appendix 
A for detail. Therefore, there is no finite interval of $x$ on which $P=Q=0$ is satisfied. Thus $k>0$ case is excluded.

Therefore, we have proved that if $\mu=$ constant, then $\dot{\zeta}=0$, namely the threebody keep its shape of the triangle being similar.

Inversely, if $\dot{\zeta}=0$, then obviously $\mu(\zeta)$ is constant. This completes a proof for the Saari's homographic conjecture for the case $\alpha=2$ planar equal-mass three-body problem.

\section{Acknowledgments}

The authors thank Richard Moeckel and Richard Montgomery for sending us their preprint on the shape variable and the Lagrangian. This research of one of the author T. Fujiwara has been supported by Grand-in-Aid for Scientific Research 23540249 JSPS.

\section{Appendix A. Details in calculation}

In this Appendix, details in calculation are shown. The following calculations were performed using Mathematica 8.0.1.0.

To eliminate the variable $y^{2}$ from the equation $P=Q=0$, we calculate the resultant of $P$ and $Q$ with respect to $y^{2}$,

$$
R=\operatorname{Resultant}\left[P, Q, y^{2}\right] \text {. }
$$

In the actual calculations, we replaced $y^{2}$ with $Y$ and calculated $\operatorname{Resultant}[P, Q, Y]$, because Mathematica doesn't accept $y^{2}$ as a variable.

Then, the coefficients $c_{n}, n=0,1,2, \ldots, 24$ in the equation (48) are polynomials of $C^{2}, k^{2}$ and $\mu_{0}$. To eliminate $k^{2}$ from the equation $c_{24}=c_{23}=0$, we again calculate the resultant of $c_{24}$ and $c_{23}$ with respect to $k^{2}$,

$$
\begin{aligned}
d_{1} & =\operatorname{Resultant}\left[c_{24}, c_{23}, k^{2}\right] \\
& =D_{1} \mu_{0}^{6}\left(\mu_{0}-1\right)^{3}\left(2 \mu_{0}-1\right)^{12}\left(\left(3 \mu_{0}-1\right) C^{2}+2 \mu_{0}\left(2 \mu_{0}-1\right)^{2}\right) C^{2},
\end{aligned}
$$

with a big integer $D_{1}$. The configurational measure $\mu$ is not smaller than 3 ,

$\mu=\frac{1}{3}\left(\sum_{i<j} r_{i j}^{2}\right)\left(\sum_{i<j} \frac{1}{r_{i j}^{2}}\right) \geq 3\left(r_{12}^{2} r_{23}^{2} r_{13}^{2}\right)^{1 / 3}\left(\frac{1}{r_{12}^{2} r_{23}^{2} r_{13}^{2}}\right)^{1 / 3}=3$.

Therefore, $\mu_{0} \geq 3$. Then, $d_{1}=0$ yields $C^{2}=0$. For $C^{2}=0$, the resultant of $c_{24}$ and $c_{22}$ with respect to $k^{2}$ is

$$
\begin{aligned}
d_{2} & =\text { Resultant }\left[c_{24}, c_{22}, k^{2}\right] \\
& =D_{2} \mu_{0}^{12}\left(\mu_{0}-1\right)^{4}\left(2 \mu_{0}-1\right)^{16} \neq 0 .
\end{aligned}
$$

Where, $D_{2}$ is another big integer. Therefore, it is impossible to make $c_{24}=c_{23}=$ $c_{22}=0$ simultaniously.

This completes a proof that there is no parameter $C^{2}, k^{2}$ and $\mu_{0}$ to make $R=0$ identically.

\section{Appendix B. Properties of the shape variable}

In this appendix, some properties of the shape variable $\zeta$ is shown. 


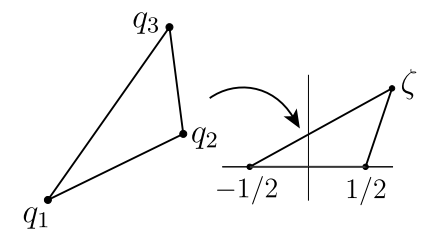

Figure B1. The definition of shape variable $\zeta$. A transformation keeping similarity and the orientation transforms the triangle $q_{1} q_{2} q_{3}$ to the triangle $a b \zeta$ with $a=-1 / 2, b=1 / 2$.

\section{Appendix B.1. Geometrical interpretation of the shape variable}

According to the work of Moeckel and Montgomery [ $\underline{6}$, we defined the shape variable $\zeta$ as the ratio of the Jacobi coordinates, in the section 11. Here, we give a geometrical interpretation of this definition.

For given triangle $q_{1} q_{2} q_{3}$, we can transform the points $q_{1} \mapsto a=-1 / 2$ and $q_{2} \mapsto b=1 / 2$ keeping the similarity and the orientation of the triangle. The points $a$ and $b$ are fixed. Let $\zeta$ be the image of $q_{3}$ by this transformation. So, the triangles $q_{1} q_{2} q_{3}$ and $a b \zeta$ are similar and have same orientation. See figure B1. It is clear that the variable $\zeta$ describe the shape of the triangle $q_{1} q_{2} q_{3}$. This gives an alternative definition of the shape variable $\zeta$.

The center of mass of the triangle $a b \zeta$ is $\zeta / 3$. To make the center of mass being fixed to the origin, we subtract $\zeta / 3$ from the three vertices. Thus, we have three vertices $\xi_{1}=-1 / 2-\zeta / 3, \xi_{2}=1 / 2-\zeta / 3$ and $\xi_{3}=2 \zeta / 3$. These are the equations (8) $-(10)$.

\section{Appendix B.2. Contribution to the angular momentum of the shape change}

The motion in $\zeta$ contributes to a size change and the angular momentum as well as shape change. To eliminate the contribution to the size change, we normalized $\xi_{k}$ by the size $\sqrt{\sum_{l}\left|\xi_{\ell}\right|^{2}}$. Let us write the normalized variable $\eta_{k}$,

$$
\eta_{k}=\frac{\xi_{k}}{\sqrt{\sum_{l}\left|\xi_{\ell}\right|^{2}}}
$$

Then, $\eta_{k}$ have unit size, therefore, have no contribution to the size change.

The variables $\eta_{k}$ still contribute to the angular momentum. The equation (18) describes that the total angular momentum is the sum of the angular momentum of the rotation angle $I \dot{\theta}$ and the angular momentum of the shape change $I \sum_{k} \eta_{k} \wedge \dot{\eta}_{k}$.

We can subtract the angular momentum from $\eta_{k}$. The result is the variable $Q_{k}$, $k=1,2,3$ introduced by Diacu et al. They defined $Q_{k}$ as follows,

$$
Q_{k}(t)=\exp \left(-i \int_{0}^{t} \frac{C d t}{I}\right) \frac{q_{k}}{\sqrt{I}} .
$$

They showed that $Q_{k}$ satisfy $\sum_{k} Q_{k}=0, \sum_{k}\left|Q_{k}\right|^{2}=1$ and $\sum_{k} Q_{k} \wedge \dot{Q}_{k}=0$ 3. Namely, the variables $Q_{k}$ have unit size and no rotation (vanishing angular momentum). They used this variables $Q_{k}$ to analyze the shape change.

To have a expression for $Q_{k}$ by $\zeta$, let us write the relation between $\zeta$ and $q_{k}$. Integrating the equation (18), we get the expression for $\theta(t)$,

$$
\theta(t)=\int_{0}^{t} \frac{C d t}{I}+\theta(0)-\frac{2}{3} \int_{0}^{t} \frac{\zeta \wedge \dot{\zeta} d t}{\frac{1}{2}+\frac{2}{3}|\zeta|^{2}}
$$


Substituting this expression to the equation (11), we get the relation,

$$
q_{k}=\sqrt{I} \exp \left(i \int_{0}^{t} \frac{C d t}{I}+i \theta(0)-\frac{2 i}{3} \int_{0}^{t} \frac{\zeta \wedge \dot{\zeta} d t}{\frac{1}{2}+\frac{2}{3}|\zeta|^{2}}\right) \frac{\xi_{k}}{\sqrt{\frac{1}{2}+\frac{2}{3}|\zeta|^{2}}}
$$

with the equations (8)-10 for $\xi_{k}$. Now, we can express $Q_{k}$ by $\zeta$,

$$
Q_{k}=\exp \left(i \theta(0)-\frac{2 i}{3} \int_{0}^{t} \frac{\zeta \wedge \dot{\zeta} d t}{\frac{1}{2}+\frac{2}{3}|\zeta|^{2}}\right) \frac{\xi_{k}}{\sqrt{\frac{1}{2}+\frac{2}{3}|\zeta|^{2}}} .
$$

Note that the mutual relations between $q_{k}(t), Q_{k}(t)$ and $\zeta(t)$ are not local in time. For example, the variable $Q_{k}(t)$ depend on the history of $\zeta\left(t^{\prime}\right)$ with $t \geq t^{\prime} \geq 0$.

\section{Appendix B.3. Energy for the shape change}

The term "energy for the shape change" should be treated carefully. In the equation 19, it is natural to understand the therm $\dot{I}^{2} /(8 I)$ be the energy for the size change and $C^{2} /(2 I)$ be the energy for the rotaion. The last term should be the "energy for the shape change". This is not the kinetic energy of $\eta_{k}, I / 2 \sum_{k}\left|\dot{\eta}_{k}\right|^{2}$. Because this term contains the rotation energy $I / 2\left(\sum_{k} \eta_{k} \wedge \dot{\eta}_{k}\right)^{2}$, which is counted in the energy for the rotation $C^{2} /(2 I)$. In the equation $\left.\sqrt{12}\right)$, the "energy for the shape change" is naturally defined by the kinetic energy minus the rotation energy,

$$
\frac{I}{2} \sum_{k}\left|\dot{\eta}_{k}\right|^{2}-\frac{I}{2}\left(\sum_{k} \eta_{k} \wedge \dot{\eta}_{k}\right)^{2}=\frac{I}{6} \frac{|\dot{\zeta}|^{2}}{\left(\frac{1}{2}+\frac{2}{3}|\zeta|^{2}\right)^{2}} .
$$

One may be convinced of this result by knowing that this is equal to $I / 2 \sum_{k}\left|\dot{Q}_{k}\right|^{2}$, the kinetic energy for $Q_{k}$ which have unit size and no rotation. To see this, an alternative expression for B.5

$$
Q_{k}=\exp \left(i \theta(0)-i \int_{0}^{t} \sum_{l} \eta_{\ell} \wedge \dot{\eta}_{\ell} d t\right) \eta_{k}
$$

will be useful.

\section{References}

[1] Alain Chenciner, Introduction to the $N$-body problem, Preprint http://www.bdl.fr/Equipes/ASD/preprints/prep.1997/Ravello.1997.pdf 1997

[2] Alain Chenciner, Some facts and more questions about the Eight, Topological Methods, Variational Methods and Their Applications, Proc. ICM Satellite Conf. on Nonlinear Functional Analysis (Taiyuan, China, 1418 August 2002) (Singapore: World Scientific) pp $77-88,2003$

[3] Florin Diacu, Toshiaki Fujiwara, Ernesto Pérez-Chavela and Manuele Santoprete, Saari's homographic conjecture of the three-body problem, Transactions of the American Mathematical Society, 360, 12, 6447-6473, 2008.

[4] Richard Moeckel, A computer assisted proof of Saari's conjecture for the planar three-body problem, Transactions of the American Mathematical Society 357, 3105-3117, 2005.

[5] Richard Moeckel, Saari's conjecture in $\mathbb{R}^{4}$, Presentation at Saarifest 2005, April 7, 2005, Guanajuato, Mexio.

[6] Richard Moeckel and Richard Montgomery, Lagrangian reduction, regularization and blow-up of the planar three-body problem, preprint, 2007.

[7] Gareth E. Roberts, Some counterexamples to a generalized Saaris conjecture, Transactions of the American Mathematical Society, 358, 251-265, 2006. 
[8] Donald Saari, On bounded solutions of the n-body problem, Periodic Orbits, Stability and Resonances, G.E.O., Giacaglia (Ed.), D. Riedel, Dordrecht, 76-81, 1970.

[9] Donald Saari, Collisions, rings, and other Newtonian N-body problems, American Mathematical Society, Rigional Conference Series in Mathematics, No. 104, Providence, RI, 2005.

[10] Donald Saari, Some ideas about the future of Celestial Mechanics, Presentation at Saarifest 2005, April 8, 2005, Guanajuato, Mexio. 